\title{
Design and Validation of a Mobile Robot for Power Line Inspection and Maintenance
}

\author{
Serge Montambault ${ }^{1}$ and Nicolas Pouliot ${ }^{2}$ \\ Hydro-Québec Research Institute (IREQ) 1740 Lionel-Boulet Blvd, Varennes \\ Québec, Canada, J3X 1S1, ${ }^{1}$ montambault.serge@ireq.ca, ${ }^{2}$ pouliot.nicolas@ireq.ca
}

\begin{abstract}
Summary. This paper presents the LineScout Technology, a mobile teleoperated robot for power line inspection and maintenance. Optimizing several geometric parameters achieved a compact design that was successfully tested over many line configurations and obstacle sequences. An overview of the technology is presented, including a description of the control strategy, followed by a section focusing on key aspects of the prototype thorough validation. Working on live lines, up to $735 \mathrm{kV}$ and 1,000 A, means that the technology must be robust to electromagnetic interference. The third generation prototype, tested in laboratory and in field conditions, is now ready to undertake inspection pilot projects.
\end{abstract}

\section{Introduction}

Power line inspection and maintenance activities have lagged behind in the use of robotic technologies even though power line maintenance work must be carried out routinely in hostile environments and hard-to-reach locations. Working on energized lines is now a necessity for most maintenance jobs. Transmission grid owners are looking towards innovation as transmission systems are under pressure from increasing loads and stringent reliability requirements [1-2].

Although some tasks have been successfully automated [3] and more research teams have been active in mobile robotics in recent years [4-5], most research results published in the past are based on concepts or prototype technologies that never made it onto transmission networks [6-7].

Following an extensive review of technologies applied to transmission line inspection and maintenance [8], the authors undertook the development of LineScout Technology as part of a Hydro-Québec research program that aims at providing robotic technologies to perform inspection and maintenance on its transmission lines [9]. From the very beginning, the key objective was to design a robust, reliable and field oriented mobile platform that would be applicable to real inspection and maintenance tasks, aligned with power line maintenance teams' considerations. 


\section{System Requirements}

A complete review of transmission line components and circuit configurations was conducted in order to identify the wide variety of obstacles that must be cleared by the robot. Among these, warning spheres (0.76-m diameter, Fig. 1A) and corona rings (Fig. 1B) were chosen as the longest obstacle to cross. Other examples of critical components identified are double insulator string configurations and vibration dampers potentially damaged by excessive vibration (Fig. 1C, 1D).
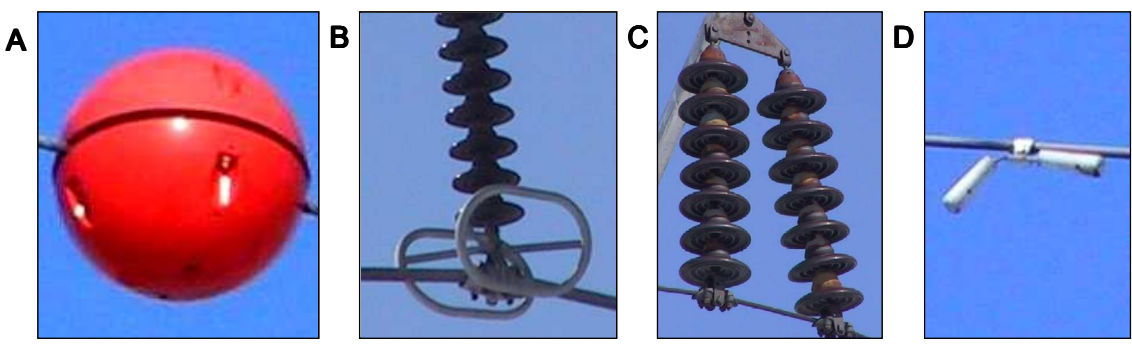

Fig. 1. Identified obstacles

Since inspection must be performed on energized lines, one of the most important requirements, besides being robust to electromagnetic interference (EMI), is to keep the size and weight of the platform to a strict minimum in order to maintain a safe dielectric distance from ground and other circuits. Table 1 shows other constraints and target values.

Table 1. Design requirements

\begin{tabular}{ll}
\hline Line components & Value \\
\hline Conductor or splice diameter & $12-85 \mathrm{~mm}$ \\
Maximum obstacle length & $0.76 \mathrm{~m}$ \\
Maximum conductor temperature & $95.0^{\circ} \mathrm{C}$ \\
Number of conductors & $1-4$ \\
Maximum slope in span & $30^{\circ}$ \\
Ambient operating temperature & $0^{\circ} \mathrm{C}-40^{\circ} \mathrm{C}$ \\
\hline Platform & \\
\hline Weight & $100 \mathrm{~kg}$ \\
Length x Height & $1.37 \mathrm{~m} \mathrm{x} 0.75 \mathrm{~m}$ \\
Traction force & $500 \mathrm{~N}$ \\
Linear speed & $1.0 \mathrm{~m} / \mathrm{s}$ \\
Battery life & $5.0 \mathrm{hours}$ \\
Communication signal range & $5.0 \mathrm{~km}$ \\
EMI robustness & $735 \mathrm{kV}, 1000 \mathrm{~A}$ \\
\hline
\end{tabular}


Design and Validation of a Mobile Robot

For Power Line Inspection and Maintenance

\section{Design of the Technology}

\subsection{Mechanical Concept}

Using one conductor as a support, rolling with wheels for locomotion not only allows moving quickly and efficiently along the power line, but also makes it possible to roll over some obstacles (compression splices, vibration dampers). To clear other types of obstacles, the approach chosen uses the sequence schematized in Fig. 2.

LineScout is built around three independent frames (Fig. 2): the wheel frame (dark frame), which includes two motorized rubber wheels called "traction wheels", the arm frame (light frame), with two arms and two grippers, and the center frame (white circle), which links together the first two frames (called "extremity frames") and allows them to slide and pivot. As an obstacle is reached, the arm frame is deployed so that the two arms and grippers can temporarily support the robot while the wheel frame is transferred on the other side of the obstacle. To do so, the wheels themselves are flipped down under the obstacle.

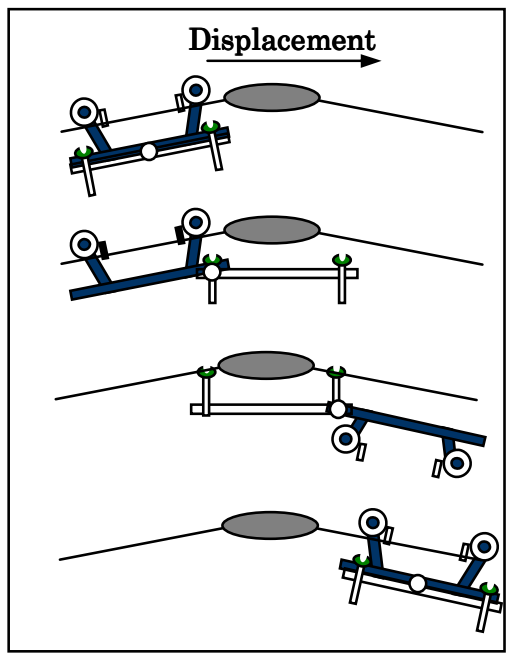

Fig. 2. Obstacle-clearing sequence

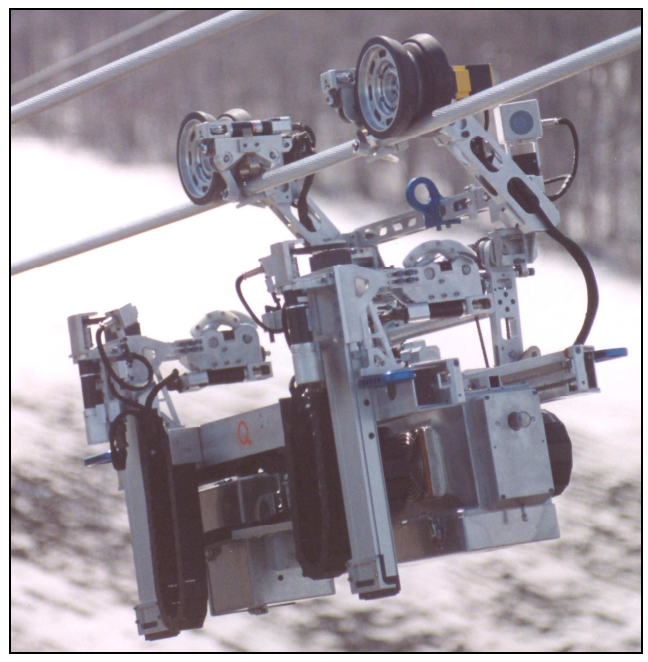

Fig. 3. The LineScout mobile platform

The development of a solution based on the approach in Fig. 2 resulted in the prototype shown in Fig. 3. Besides the traction wheels, the wheel frame includes a camera mounted on a pan-and-tilt unit as well as a pair of safety rollers for platform stability when crossing obstacles. The center frame supports the electronics cabinet and battery pack. Finally, it is the arm frame, which includes two other cameras on pan-and-tilt units, that supports most of the application modules mounted on the mobile platform.

The center frame is a strategic system of the mobile platform since it generates the movement of the two other frames by sliding them in opposite directions, while supporting about $40 \%$ of the platform's weight. By doing so, a gradual 
weight transfer is obtained during the obstacle clearance sequence and this greatly minimizes the cantilevered load applied when extremity frames are in total extension, as in Fig. 4. Therefore, the center frame has to be stiff for predictable platform behavior yet flexible enough to adapt to particular situations, like a change in direction of the conductor at a suspension clamp. Some amount of passive flexibility between the frames was therefore maintained.

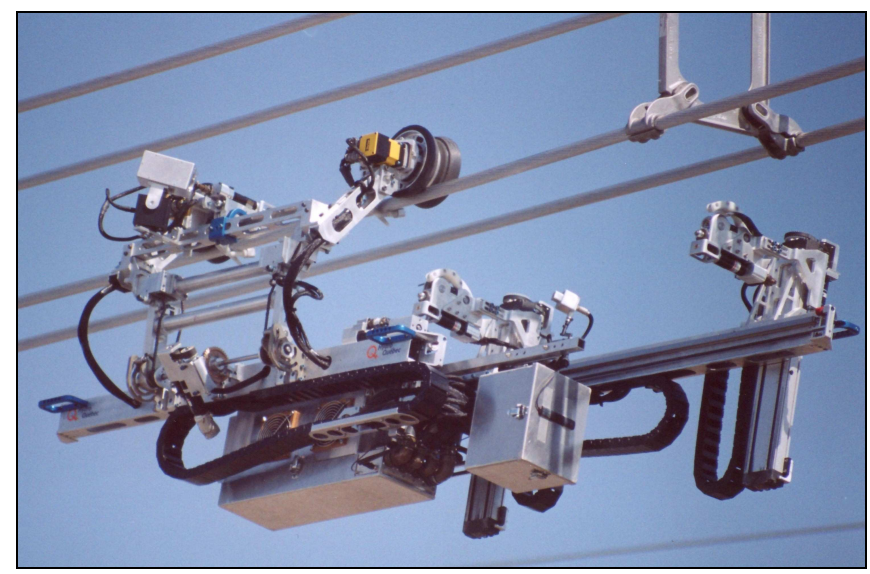

Fig. 4. LineScout about to clear a spacer damper

\subsection{Geometrical Optimization}

One of the main advantages of this concept in comparison to other existing prototype is that it is neither limited to a specific size of obstacle nor to a specific distance between adjacent obstacles, making it versatile and adaptable to various sequence of obstacles. In fact, six different clearing sequences were identified as strategies for crossing consecutive obstacles, depending on their sizes and the distance between them. Three of these variants are presented in Fig. 5. Also on this figure, Lomax is defined as the maximum length of a crossable obstacle, and Domin is defined as the minimum distance between two consecutive obstacles.

In order to optimize the LineScout geometry according to the range of expected possibilities, eight key parameters were defined, as summarized by Table 2 and Fig. 6. Values of the critical geometric parameters of LineScout have then been optimized based the six possible sequences for clearing line component configurations and based on the expected relation between Lomax and Domin.

This optimization process assures that at least one clearing sequence is usable for any given situation. Another key advantage of this optimization is the resulting relative compactness: a vehicle with an overall length of only $1.37 \mathrm{~m}$ is able to clear obstacles $0.76 \mathrm{~m}$ in diameter. This design still allows a relatively long $(0.79 \mathrm{~m})$ wheelbase making the vehicle very stable and able to carry a larger payload. 
Design and Validation of a Mobile Robot For Power Line Inspection and Maintenance

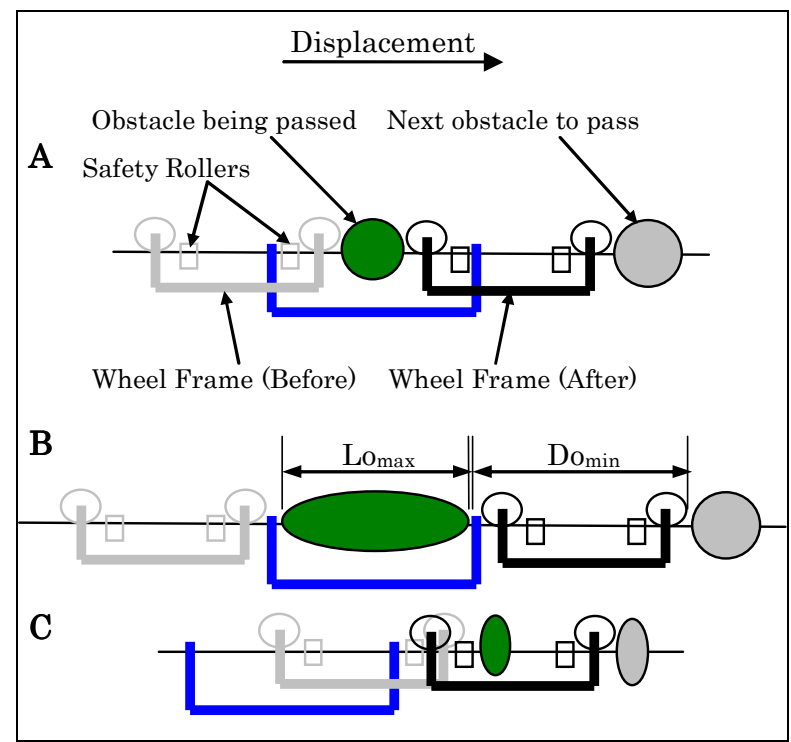

Fig. 5. Examples of clearing sequences

Table 2. Critical geometric parameters

\begin{tabular}{lc}
\hline \multicolumn{1}{c}{ Parameter description } & Symbol \\
\hline External diameter of the traction wheels & $\mathrm{Dt}$ \\
Wheelbase of the traction wheels & $\mathrm{Et}$ \\
Wheelbase of the safety rollers & $\mathrm{Er}$ \\
Wheelbase of the grippers & $\mathrm{Ea}$ \\
Distance from conductor to center of rotation & $\mathrm{Lz}$ \\
Translation range of movement & $\mathrm{Tmax}$ \\
Rotation range of movement & $\mathrm{Rmax}$ \\
Grippers' range of movement & $\mathrm{Amax}$ \\
\hline
\end{tabular}

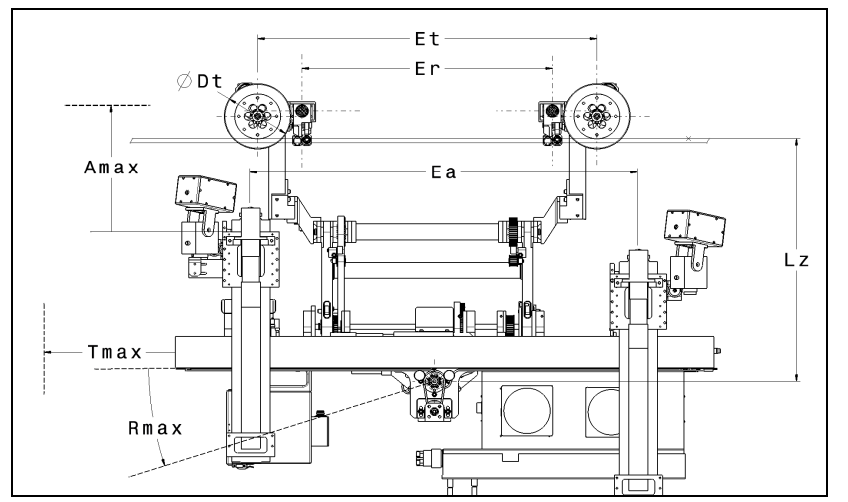

Fig. 6. Critical parameters of the mobile platform 


\subsection{Electronics and Control}

An overview of the onboard electronics is presented in Fig. 7A. The electronics cabinet holds the onboard computing system which includes 19 custom boards using PIC microcontroller technology. These boards are dedicated to motion control (wheel motors, motion motors, and pan-and-tilt motors), DC-DC converters, communication systems (data and video), protection circuits, video image management and data acquisition. Also, $12-\mathrm{V}, 24-\mathrm{V}$ and $48-\mathrm{V}$ buses carry power to the boards and components. An RS-485 bus is used for communication between the boards.

The data communication system operates at $900 \mathrm{MHz}$ (spread spectrum technology) while the video transmission system operates at $2.4 \mathrm{GHz}$. Even though harvesting power from the powerline is technically feasible, Lithium-Ions battery pack that allows for full day autonomy was a simpler, more reliable, and much lighter choice.

All seventeen motors (counting the pan-and-tilt units) and their encoders and brakes, plus all cameras and sensors are linked to the central electronics cabinet by a total of $30 \mathrm{~m}$ of double-shielded cables that are securely coupled by military-type connectors.

A

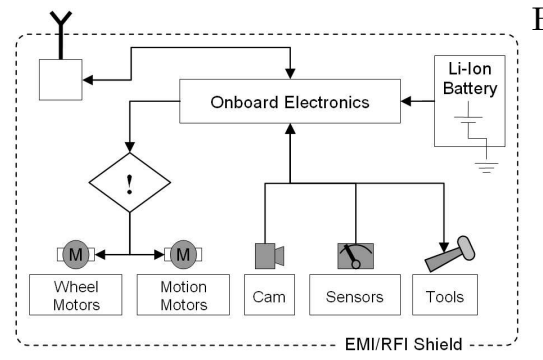

$\mathrm{B}$

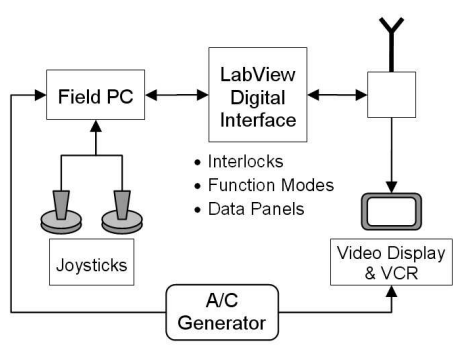

Fig. 7. Onboard electronics and Ground Control Unit schematics

The ground control unit (Fig. 7B) consists of a portable computer (military standards) with touch screen interface and two industrial three-axis joysticks equipped with five push buttons. Control software was developed on LabView software. A video display unit allows images from the mobile platform to be viewed for control of the platform and archiving of inspection images.

One of the determinant decisions taken at the beginning of the project was to make the LineScout a teleoperated technology, instead of an autonomous vehicle. Of course it had to be as intuitive to operate as possible, but its initial purpose is more or less to extend the reach of experienced linemen, allowing them to safely inspect and maintain the network that they know well. This is another key difference between this technology and previous ones and this is likely to facilitate its introduction into service.

The strategy adopted to control the mobile platform and allow a human operator to handle so many axis of movement with two joysticks is based on the notion of modes. Modes are based on a series of actions relevant to a particular se- 
quence of movement. In displacement mode, for instance, only the traction wheel motors and safety roller motors can be actuated by the operator by mean of the actuation joystick, the other joystick being always dedicated to the control of cameras and pan-and-tilt units.

When a mode change is necessary, interlocks manage the transition to make sure it follows a safe and reliable sequence of operations. Most motor movements are speed-controlled and limited through a mixed strategy based on position feedback and a peak current limit reached as the actuated axis approaches a mechanical limit.

A watchdog (represented by an exclamation mark on Fig. 7A) reduces power to the motors automatically if unexpected commands are received or loss of transmission suddenly occurs. A hardware emergency stop, located near the joysticks, can also activate the watchdog. What has been defined as "sleep mode" allows minimal energy consumption, all systems being down except for data communications.

The operator receives feedback mainly from the three cameras. Two-axis inclinometers that return the platform pitch and roll in absolute values along with each motor position feedback are also used to animate a 3D model of the plateform in a separate window of the graphical interface.

Even though the current control strategy only requires so few sensors, plans are laid out to progressively increase the level of automation and the onboard electronics allow for additions as they would seem necessary. Obstacle detection and identification will eventually allow autonomous selection of the appropriate clearing sequence. Instantaneous weight-on-wheel measurement, Global Positioning System (GPS), and thermal measurement are also examples of potential short-term additions.

\subsection{Implemented Application Modules}

A total of four controllable cameras can be used to give very flexible and complementary points of view for visual inspection of line components, such as cylindrical warning markers, suspension clamps and insulator strings as they are being passed. Splice condition evaluation based on measuring the electrical resistance of the splice is an application readily implemented on LineScout Technology. A motorized system was developed for positioning an off-the-shelf sensor used to collect information on the compression splice's electrical resistance. Development was recently completed of a module to temporarily repair broken conductor strands by installing a copper clamp close to the damaged area. In addition to collecting visual information for better repair job planning, this module eliminates the risk of further deterioration of the conductor as broken strands unravel under windy conditions.

\section{Validation}

A systematic approach was adopted from day one to ensure the technology's reliability and safe operation. After three years of development and testing, a 
validated, field-oriented prototype is ready to undertake inspection pilot projects onto strategic power utility equipments.

Validation included lab testing (software simulation, test beds for subsystems, testing on a power line mockup, live-line testing, demos and training) and field testing (IREQ's experimental power line, de-energized lines, energized lines and pilot projects).

\subsection{Failure Mode and Effect Analysis}

Literature [10] suggests approaches to assess the reliability of systems being designed. The starting point of our analysis was to identify undesirable events and their consequences on the powerline system (component integrity, service continuity, worker safety and public safety) and on the robot itself. Most probable causes of these events were then systematically identified as all subsystems of the mobile platform were broken down into their simplest parts.

Failures of previous generations of the mobile platform were classified into three sources: mechanical, electronics and control/software. With the firstgeneration prototype, most failures $(80 \%)$ came from their mechanics and electronics: harness and connectors, belt transmission systems, motor shaft couplings, motor amplifier controllers, communication system and electric brakes. Solutions have since been applied for each weak point identified.

The latest generation of the platform is used to implement fault detection and recovery, addressing electronics and control failures or the most probable cause of potential failures. Live-line testing, which is a completely different context for experiments, pointed to failures of communication system components, harness grounds and shields as well as electronic protection circuitry.

Another key benefit of this exercise was to provide a detailed maintenance procedure and a pre-installation verification list.

\subsection{Endurance testing}

For over two years, the mobile platform has been tested under indoor and outdoor simulations of maintenance operations. Throughout these tests, failures, operator mistakes and surprising behaviors were logged and subsequently solved. These tests were conducted mainly to observe any mechanical system premature wear, battery performance, board and component temperature rise, overall design performance, electronic board and component behavior under peak power draw, cable routing, actuator choices, structure strength and flexibility, additional feedback required and PID control loop gain adjustment.

The mobile platform rolled over $75 \mathrm{~km}$ of conductors and crossed 700 obstacles. The experience gained from this endurance testing was introduced into the design of second and third generation prototypes.

Traction force measurements on various slope conditions and stability evaluation were conducted. Cold-temperature operation of LineScout was also validated after the robot spent the entire night outside at $-13^{\circ} \mathrm{C}$ and functioned normally. 
Design and Validation of a Mobile Robot

For Power Line Inspection and Maintenance

\subsection{EMI Robustness}

The electromagnetic environment surrounding a live $735-\mathrm{kV}$ line with an average load of 1,000 $\mathrm{A}$ is quite a challenge for electronics that operate at voltage between $12 \mathrm{~V}$ and $48 \mathrm{~V}$. Boards were designed carefully respecting basic principles of EMI robustness. Since the entire structure must conduct electricity to minimize any difference in potential developed across components, particular care was taken in designing high-quality shields (connectors, harness, electronics cabinet, battery and peripheral systems). The traction wheels are made out of a conductive natural rubber specially developed and molded for this application. All other components in contact with the energized conductor, such as the safety rollers and grippers, are covered by a protective sleeve made of soft conductive nylon that does not scar or dent conductors.

An incremental testing strategy was adopted regarding electromagnetic immunity of the systems. Hydro-Quebec Research Institute (IREQ) expertise in this field made it possible to quickly focus on key issues and design robust onboard electronics. A high-voltage test chamber was then used to generate the electromagnetic equivalent of a 500-kV transmission line. On Fig. 8, the live line installation of the LineScout is simulated as an electric arc is applied for $20 \mathrm{sec}^{-}$ onds on different parts of the robot (mechanical systems, harness and cameras). Areas with strong corona emissions were identified using a UV camera and mechanical parts were modified accordingly. LineScout was then tested on a conductor with a 1,000 A load, submitting its electronics to a strong magnetic field.

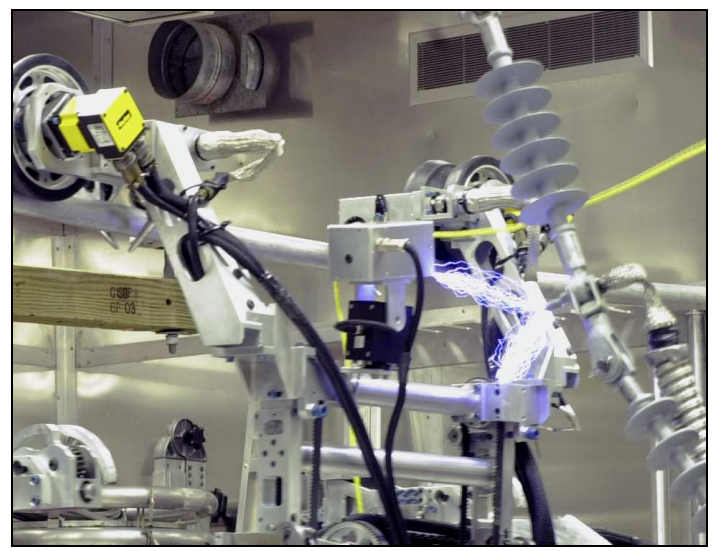

Fig. 8. LineScout being tested at $500 \mathrm{kV}$ in IREQ's facility

The field tests for in situ validation on energized power lines were conducted on a live $315-\mathrm{kV}$ line. After successful testing, the mobile platform was finally tested on a live $735-\mathrm{kV}$ line.

\section{Conclusion and Future Work}

A mobile robotic platform able to cross most of the obstacles found on transmission lines has been presented, for which a patent application was filed in 2004 
[11]. The third-generation prototype has been tested and validated under field conditions. Several features of the technology make it distinct with respect to previous attempts to incorporate robotic into the field of power utilities. Among them is the reasonable payload it can carry, its versatility to adapt to unforeseen sequence of obstacle, the intuitive teleoperation type of control, its reliability, and the capacity to add sensors and tools in a modular way.

Obstacle detection and identification will eventually allow autonomous selection of the appropriate strategy for crossing obstacles. Another high-value application for LineScout Technology is visual inspection of line components that are found on conductor bundles. The robotic arm needed for this task, under development, will also lend itself to splice condition evaluation and other live-line work.

\section{References}

1. Montambault S, Pouliot N (2004) On the Economic and Strategic Impact of Robotics Applied to Transmission Line Maintenance, 7th International Conference on Live Maintenance (ICOLIM 2004), Bucharest, Romania

2. Jiang B, Mamishev V (2004) Robotic monitoring of power systems, IEEE Transactions on Power Delivery, Vol. 19,No. 3

3. Campos M et al (2002) A Mobile Manipulator for Installation and Removal of Aircraft Warning Spheres on Aerial Power Transmission Lines, Universidade Federal de Minas Gerais, 31270-901, Belo Horizonte, MG, Brazil

4. Zhou F Y, Wang J D, Li Y B, Wang J, Xiao H R (2005) Control of an inspection robot for $110 \mathrm{kV}$ power transmission lines based on expert system design methods, Proceedings of the 2005 IEEE Conference on Control Applications, Toronto, Canada

5. Rochia J, Sequeira J (2004) The development of a robotic system for maintenance and inspection of power lines, Proceedings of the 35th International Symposium on Robotics, Paris

6. Earp G (1996) EA Technology's Robotics Program, Proceedings of Robotics for use in the Electricity Industry

7. Sawada J et al. (1991) A Mobile Robot for Inspection of Power Transmission Lines, IEEE Transactions on Power Delivery, Vol.6, No.1, pp. 309-315

8. Montambault S, Pouliot N, (2003) The HQ LineROVer: Contributing to Innovation in Transmission Line Maintenance, 10th International Conference on Transmission and Distribution Construction, Operation and LiveLine Maintenance (ESMO 2003)

9. Montambault S, Pouliot N (2006) LineScout Technology: Development of an Inspection Robot Capable of Clearing Obstacles While Operating on a Live Line, 11th International Conference on Transmission and Distribution Construction, Operation and Live-Line Maintenance -ESMO 2006

10. Villemeur A (1988) Sûreté de fonctionnement des systèmes industriels, Editions Eyrolles, ISBN\# 2212016158

11. Montambault S et al. (2005) Remote-Controlled Vehicle Designed to be Mounted on a Support and Capable of Clearing an Obstacle, Patent Application, WO2005/101600 